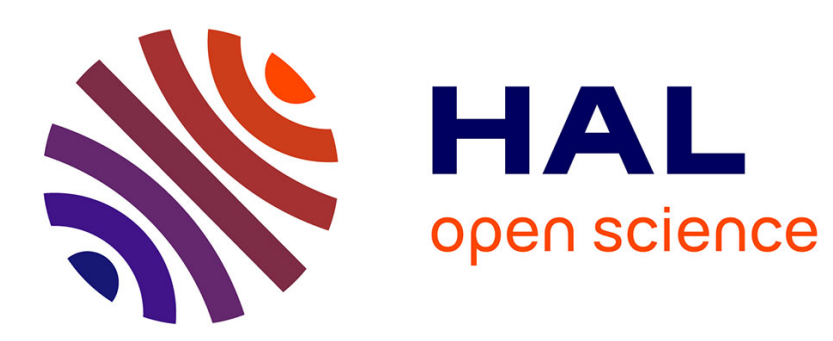

\title{
Caméra à balayage de fente et métrologie picoseconde des impulsions de lasers fonctionnant à des taux de répétition élevés
}

\author{
P. Geist, F. Heisel, A. Martz, J.A. Miehé
}

\section{> To cite this version:}

P. Geist, F. Heisel, A. Martz, J.A. Miehé. Caméra à balayage de fente et métrologie picoseconde des impulsions de lasers fonctionnant à des taux de répétition élevés. Revue de Physique Appliquée, 1984, 19 (8), pp.619-629. 10.1051/rphysap:01984001908061900 . jpa-00245230

HAL Id: jpa-00245230

https://hal.science/jpa-00245230

Submitted on 1 Jan 1984

HAL is a multi-disciplinary open access archive for the deposit and dissemination of scientific research documents, whether they are published or not. The documents may come from teaching and research institutions in France or abroad, or from public or private research centers.
L'archive ouverte pluridisciplinaire HAL, est destinée au dépôt et à la diffusion de documents scientifiques de niveau recherche, publiés ou non, émanant des établissements d'enseignement et de recherche français ou étrangers, des laboratoires publics ou privés. 


\title{
Caméra à balayage de fente et métrologie picoseconde des impulsions de lasers fonctionnant à des taux de répétition élevés
}

\author{
P. Geist, F. Heisel, A. Martz et J. A. Miehé \\ Centre de Recherches Nucléaires et Université Louis Pasteur, \\ Laboratoire de Physique des Rayonnements et Electronique Nucléaire, 23, rue du Loess, \\ 67037 Strasbourg Cedex, France
}

(Reçu le 27 février 1984, révisé le 2 mai 1984, accepté le 30 mai 1984)

\begin{abstract}
Résumé. - Les caractéristiques de différents montages expérimentaux comprenant une caméra à balayage de fente associée à un laser à colorant fonctionnant en régime de pompage synchrone ou à un laser répétitif à modes couplés et déclenché sont décrites. Les performances temporelles obtenues avec des générateurs de balayage à tension sinusoïdale $(80-100 \mathrm{MHz})$ et à rampe linéaire déclenchée $(0-800 \mathrm{kHz})$ sont présentées.
\end{abstract}

\begin{abstract}
Some experimental configurations of a streak camera used with a synchronous pumped dye laser or with a repetitive mode-locked and $Q$-switched laser are described. The overall performances are discussed with respect to synchronous and triggered operating modes of the streak camera.
\end{abstract}

\section{Introduction.}

Au cours des dernières années, l'emploi de caméras à balayage de fente s'est avéré particulièrement intéressant dans les domaines des sciences expérimentales en rapport avec la détermination de l'évolution temporelle de signaux lumineux avec une résolution à l'échelle des picosecondes [1-4]. L'intérêt de ces dispositifs s'est considérablement accru lorsque la sensibilité et la dynamique de la détection ont été augmentées grâce à l'utilisation de lasers impulsionnels à taux de répétition élevé ou variable et à l'élaboration de circuits de balayage fonctionnant en mode sinusoïdal [5] ou déclenché $[6,7]$, à très faibles fluctuations temporelles $\left(\simeq 10^{-12} \mathrm{~s}\right)$.

Dans ce travail sont décrits divers montages expérimentaux utilisés en vue de l'enregistrement de la fluorescence résolue en temps de molécules excitées par des impulsions laser récurrentes de durée très brève $\left(\lessgtr 10^{-10} \mathrm{~s}\right)$. Leurs caractéristiques sont examinées en fonction des modes de synchronisation de la caméra et du laser, et leurs performances sont illustrées par des mesures de résolution instrumentale ou de constantes de temps de fluorescence. L'instabilité de la forme des impulsions - liée à la longueur de la cavité d'un laser à colorant en régime de pompage synchrone - est mise en évidence en enregistrant des traces dues à des impulsions uniques. L'influence de celle-ci sur la résolution temporelle en régime déclenché et périodique est analysée. Les résultats de mesures de durées d'impulsions par autocorrélateur sont comparés à ceux obtenus à l'aide de la caméra à balayage de fente.

\section{Caméra à balayage de fente $[1,8]$.}

Elle est constituée d'un tube de déflexion, comportant une photocathode de sensibilité S20 (EEV-P855 ou Photochron II), couplé optiquement par un objectif (Tektronix 125, $f 1,4)$ à un amplificateur de brillance de gain élevé (Emi 9694). L'analyse des traces apparaissant sur l'écran luminophore est effectuée à l'aide d'un réseau linéaire de photodiodes (Reticon RL 512C/ 17 ou RL 1024SF) [9] associé à un convertisseur de 12 bits; le couplage est réalisé à l'aide d'un objectif de grande ouverture (Fuji 50, f1,4). Le calibrage en amplitude et en temps du système est réalisé à l'aide d'un montage de Fabry-Pérot placé devant la photocathode et composé de deux miroirs de coefficient de réflexion 0,7 séparés d'une distance $l$. A une impulsion incidente unique correspond à la sortie un train d'impulsions : les intensités de deux éclairs consécutifs sont dans un rapport 2 et l'intervalle de temps qui les sépare est de $2 l / c$ ( $c$ étant la vitesse de la lumière).

La figure 1A représente schématiquement le principe de fonctionnement d'une caméra à balayage de fente. L'impulsion lumineuse incidente de section circulaire est convertie, après passage à travers une fente $F$, en 


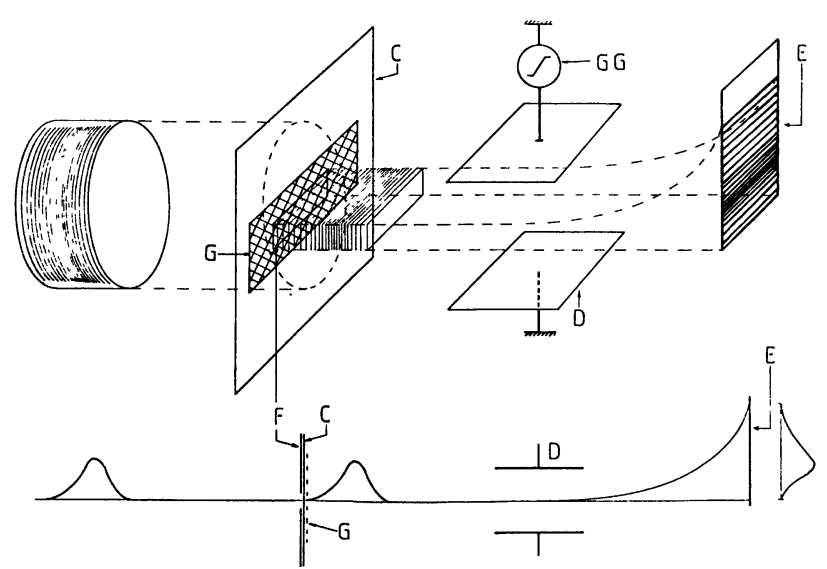

Fig. 1A. - Principe de fonctionnement d'une caméra à balayage de fente [3] : F : fente; C : photocathode; G : grille d'extraction; $\mathrm{D}$ : plaques de déviation; $\mathrm{E}$ : écran luminophore; GG : générateur sinusoïdal ou générateur de rampe.

[Schematic of a streak tube.]

un faisceau d'électrons de section rectangulaire. Après déflexion, à la distribution temporelle des instants d'arrivée des photons sur la photocathode $\mathrm{C}$, correspond une répartition spatiale de l'intensité sur l'écran luminophore $\mathrm{E}$. Les plaques de déviation $\mathrm{D}$ assurant la déflexion du faisceau d'électrons sont pilotées par des circuits de balayage donnant lieu à des sensibilités comprises entre 200 et $400 \mathrm{ps} / \mathrm{cm}$ sur l'écran luminophore. On peut distinguer essentiellement deux modes de fonctionnement de la caméra.

Lorsqu'elle est associée à un laser à colorant à modes bloqués par pompage synchrone (Spectra Physics) ou à un laser Nd:YAG continu à modes couplés (Quantronix), délivrant des impulsions avec des fréquences de répétition respectivement de 80 et $100 \mathrm{MHz}$, la déflexion est généralement assurée par une tension sinusoïdale. En effet, une méthode simple pour produire la déflexion dans une caméra consiste à appliquer une différence de potentiel sinusoïdale de fréquence égale à l'inverse de la période des impulsions laser. Une linéarité du balayage meilleure que $5 \%$ est obtenue pour une demi-amplitude de la hauteur crête à crête de la sinusoïde (Fig. 1a). Un inconvénient essentiel de ce procédé apparaît lorsque la constante de temps de décroissance de la fluorescence est voisine ou supérieure à la période de la tension de déflexion. Une seule impulsion lumineuse donne alors naissance à un signal qui est la superposition de portions de la courbe de déclin enregistrées avec un pas égal à la demi-période de la sinusoïde et avec un renversement de l'axe des temps à chaque pas (Fig. 1d). La linéarité différentielle d'un tel type de balayage est analysée en Annexe. Dans les conditions de fonctionnement du laser à colorant par décharge de la cavité (Spectra Physics) délivrant des impulsions à des taux de répétition variables $(200 \mathrm{~Hz}-800 \mathrm{kHz})$, à ce type de déflexion

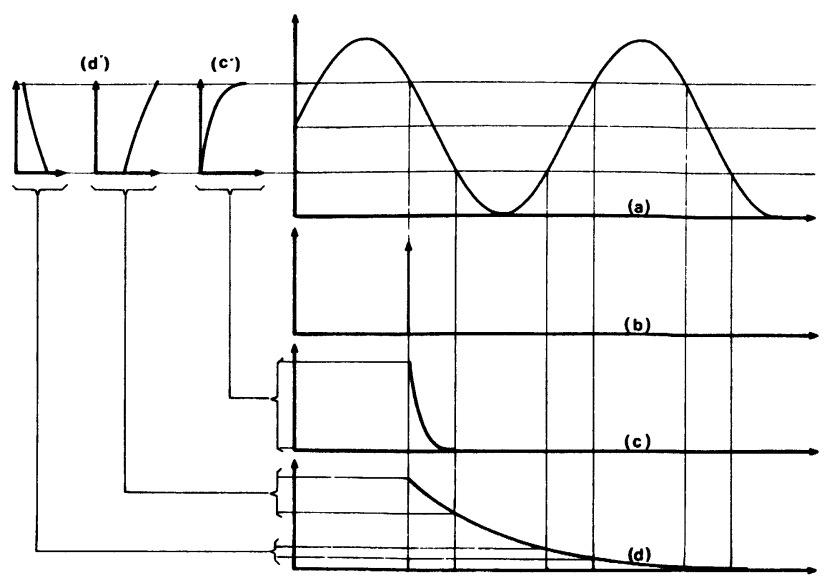

Fig. 1. - Principe de fonctionnement d'une caméra à balayage de fente à tension sinusoïdale de déflexion : (a) tension de déflexion; (b) impulsion d'excitation; (c) et (d) déclins de fluorescence de durées de vie petite et grande (voisine de la période sinusoïdale); $\left(c^{\prime}\right)$ et $\left(d^{\prime}\right)$ traces correspondantes sur l'écran luminophore.

[Synchroscan principle : (a) deflection voltage; (b) excitation pulse; (c) and (d) fluorescence decays; $\left(c^{\prime}\right)$ and $\left(d^{\prime}\right)$ corresponding streaks.]

on préfère une rampe de balayage délivrée par un générateur déclenché, constitué par des tubes microondes de puissance. Ce dispositif peut être utilisé avec le Nd:YAG fonctionnant en régime de modes couplés et déclenché.

\section{Montages expérimentaux.}

3.1 Déflexion Par tension Sinusoïdale. - Dans le dispositif expérimental représenté schématiquement sur la figure 2, le faisceau lumineux (train d'impulsions périodiques $12,5 \mathrm{~ns}$ ) issu du laser à colorant (Rh6G) à modes bloqués (DL) en phase par pompage synchrone par le laser à argon ionisé (AL) est partagé en 2 au moyen d'un miroir $(M)$. Une fraction d'environ $1 \%$ de la puissance moyenne de $120 \mathrm{~mW}$ est focalisée sur une photodiode (D) connecté à un circuit de mise en forme impulsionnelle (PS) qui délivre un signal rectangulaire périodique de fréquence $80 \mathrm{MHz}$; après amplification (A), il est appliqué à un amplificateur accordé de puissance qui constitue le circuit de déflexion de la caméra à balayage de fente (SC). Les images de l'écran correspondant aux impulsions incidentes sont numérisées et mémorisées à l'aide d'un analyseur multicanaux optique (OA) comportant le réseau de photodiodes et commandé par le circuit de contrôle (CC).

Les performances temporelles du système comprenant un tube Photochron II sont illustrées par les histogrammes de la figure 3 relevés, après réglage optimal de la longueur de la cavité du laser à colorant, pour différentes largeurs de la fente placée au voisinage de la photocathode; les profils statiques ont été 


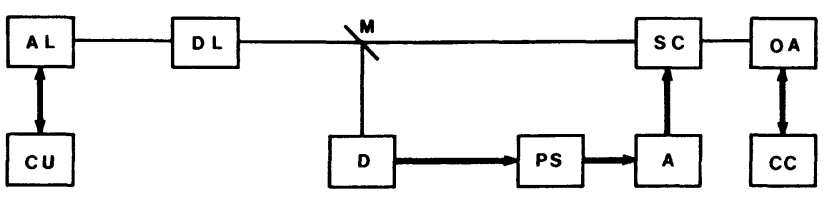

Fig. 2. - Montage expérimental utilisé pour la déflexion de la caméra par une tension sinusoïdale $(80 \mathrm{MHz})$. Synchronisation par l'intermédiaire du faisceau lumineux. $\mathrm{AL}$ : laser à argon ionisé à modes couplés (Spectra Physics 171 et 342S); DL : laser à colorant à Rh6G (S-P 375); CU : unité de contrôle (S-P 452 et 454); M : miroir; D : photodiode à semiconducteur; PS : circuit de mise en forme; A : amplificateur accordé; SC : caméra à balayage de fente; OA : analyseur multicanaux optique; $\mathrm{CC}$ : circuit de contrôle.

[Experimental set-up for synchroscan camera associated with a sync-pump dye laser (Spectra Physics).]

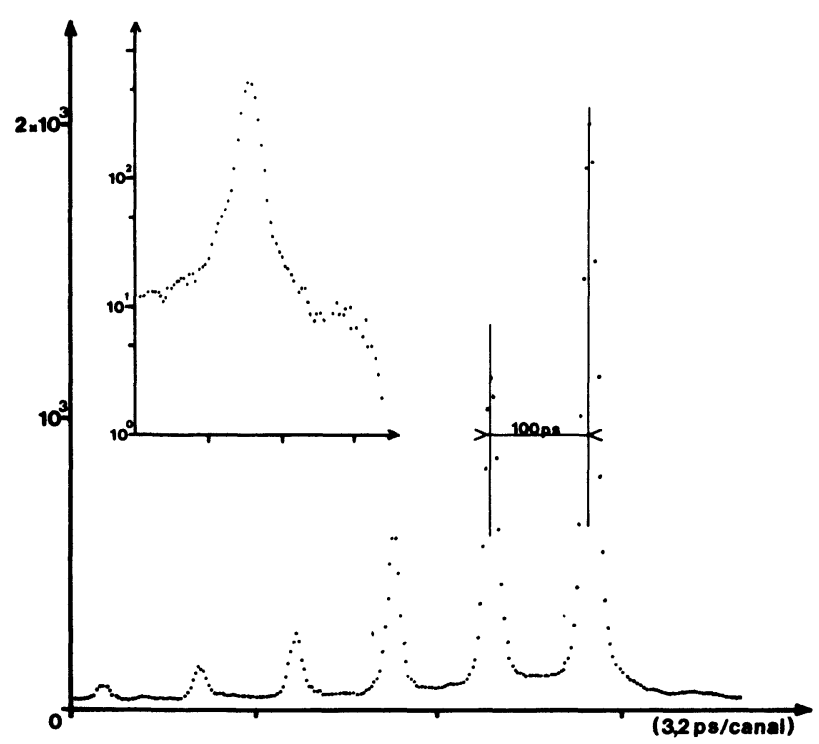

a)

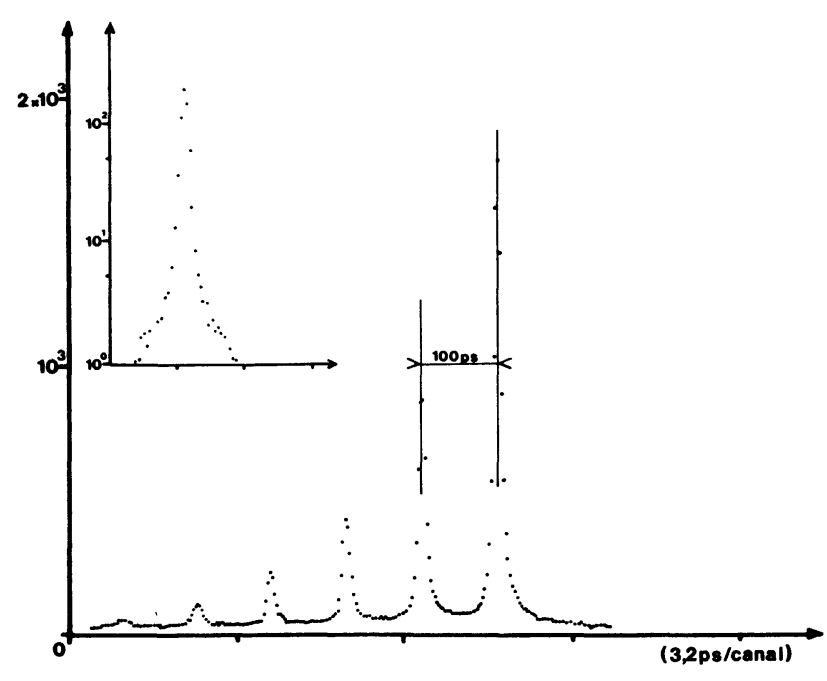

b) enregistrés et sont également indiqués - en représentation semilogarithmique - sur la même figure. $\mathrm{La}$ meilleure résolution instrumentale $l_{1 / 2}^{\mathrm{d}}=11 \mathrm{ps}$ est obtenue pour une fente de largeur à mi-hauteur totale équivalente $\left(l_{1 / 2}^{\mathrm{f}}\right)$ égale à $9 \mathrm{ps}$ correspondant à une largeur réelle d'environ $70 \mu \mathrm{m}$.

La résolution temporelle intrinsèque d'un tube à fente [10] peut être caractérisée par $\sigma_{1}=\left(\sigma_{\mathrm{K}}^{2}+\sigma_{\mathrm{s}}^{2}\right)^{1 / 2}$ où $\sigma_{\mathrm{K}}^{2}$ désigne la variance des fluctuations du temps de transit des électrons entre la photocathode et la grille, dues à la variation de leur vitesse initiale et où $\sigma_{\mathrm{s}}=(v \delta)^{-1}$ est l'écart-type de la dispersion temporelle lié à la résolution spatiale $\delta$ et à la vitesse $v$ de balayage du tube.

En supposant que les fluctuations soient de loi normale, la largeur totale à mi-hauteur $l_{1 / 2}$ de la distribution est liée à son écart-type $\sigma \operatorname{par} l_{1 / 2} \approx 2,36 \sigma$. Pour un tube Photochron II, $\delta$ est voisin de 50 paires de lignes $/ \mathrm{mm}$ et $\sigma_{\mathrm{K}}$ est estimée inférieure à $0,4 \mathrm{ps}$ pour un rayonnement de longueur $\lambda=615 \mathrm{~nm}$ [10]. Compte tenu du fait que $v=1,5 \times 10^{9} \mathrm{~cm} \mathrm{~s}^{-1}$, la résolution intrinsèque $\sigma_{1}$ est évaluée à 1 ps et sa contribution peut être négligée dans l'écart-type de la distribution expérimentale donnée par

$$
\sigma_{\exp }^{2}=\sigma_{\mathrm{f}}^{2}+\sigma_{\mathrm{i}}^{2}+\sigma_{\mathrm{e}}^{2}+\sigma_{1}^{2}
$$

où $\sigma_{\mathrm{f}}, \sigma_{\mathrm{i}}$ et $\sigma_{\mathrm{e}}$ désignent les écarts-types caractérisant la fente statique, l'impulsion lumineuse et les incertitudes de synchronisation.

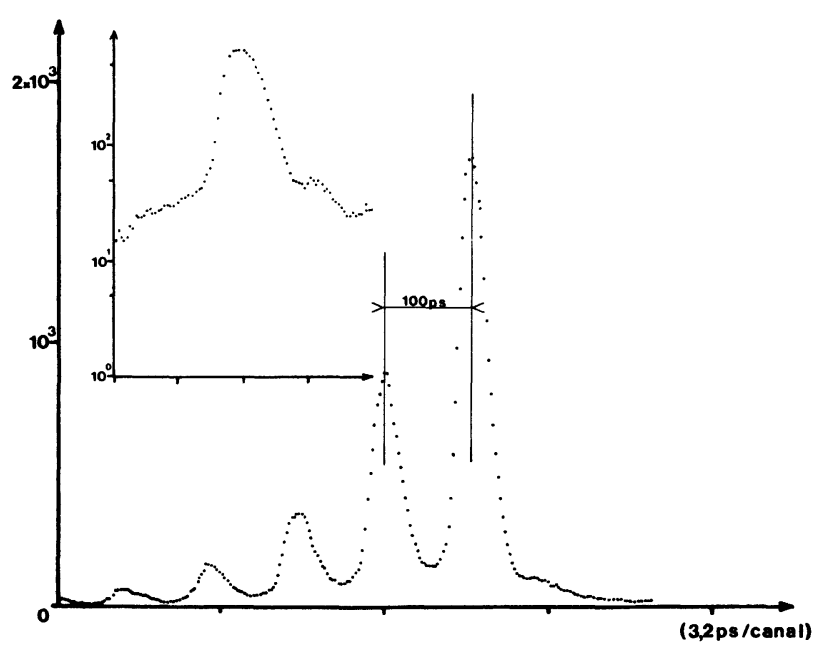

c)

Fig. 3. - Histogrammes expérimentaux relevés avec le montage de la figure 2, pour une longueur optimale de la cavité du laser à colorant et pour différentes largeurs de la fente à l'entrée de la caméra. $l_{1 / 2}^{\mathrm{f}}=$ largeur à mi-hauteur totale de la fente relevée au niveau du réseau de photodiodes $(\mathrm{OA})$, sans balayage $; l_{1 / 2}^{\mathrm{d}}=$ largeur à mi-hauteur en présence de balayage

$$
\begin{array}{ll}
\mathrm{a}: l_{1 / 2}^{\mathrm{f}}=9 \mathrm{ps}(\leftrightarrow 70 \mu \mathrm{m}), & l_{1 / 2}^{\mathrm{d}}=11 \mathrm{ps} \\
\mathrm{b}: l_{1 / 2}^{\mathrm{f}}=16 \mathrm{ps}(\leftrightarrow 125 \mu \mathrm{m}), & l_{1 / 2}^{\mathrm{d}}=19 \mathrm{ps} \\
\mathrm{c}: l_{1 / 2}^{\mathrm{f}}=47 \mathrm{ps}(\leftrightarrow 370 \mu \mathrm{m}), & l_{1 / 2}^{\mathrm{d}}=48 \mathrm{ps}
\end{array}
$$

[Experimental time resolution curves versus slit dimensions.] 
Il en résulte que $l_{1 / 2}=\sqrt{\left(l_{1 / 2}^{\mathrm{d}^{2}}-l_{1 / 2}^{\mathrm{f}^{2}}\right)}=6 \mathrm{ps}$ caractérise la contribution à la largeur de la distribution expérimentale de la durée intrinsèque du signal lumineux et de la dispersion des fluctuations temporelles introduites par le circuit de balayage. Cette dernière ne peut être évaluée que si l'on connaît la largeur des impulsions lumineuses. Pour accéder à cette information, l'enregistrement (Fig. 4) d'histo-
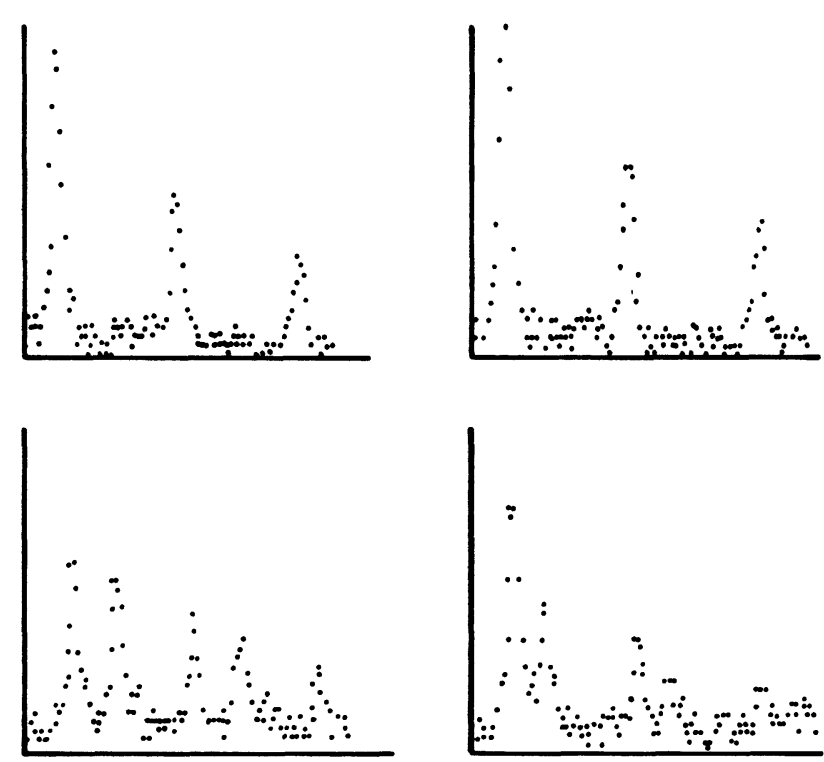

Fig. 4. - Enregistrements d'impulsions uniques, obtenus dans les conditions de réglage correspondant à la figure $3 \mathrm{a}$. [Single shots recorded with the optimal cavity length.]

grammes consécutifs à l'impact sur la photocathode d'impulsions uniques (voir $\S 3.2$ pour leur mode d'obtention) a été effectué pour le réglage correspondant à la résolution optimale observée en régime répétitif (Fig. 3a). La largeur $l_{1 / 2}(<9 \mathrm{ps})$ des courbes de la figure 4 est inférieure à celle relevée en fonctionnement récurrent, mais la présence de pré et/ou post impulsions est manifeste. Ces instabilités ne sont pas mises en évidence à l'aide de mesures par un autocorrélateur qui donne une figure stable correspondant à des impulsions lumineuses unimodales (cusp shaped autocorrelation figure) de largeur voisine de quelques picosecondes. De ces observations, il découle que les fluctuations temporelles introduites pas l'électronique de balayage doivent être négligeables et que l'élargissement observé $\left(l_{1 / 2}^{\mathrm{d}} \approx 11 \mathrm{ps}\right)$ est dû essentiellement aux variations de la forme des impulsions du laser. Ces résultats sont en accord avec ceux d'une étude de l'influence de la longueur de cavité sur la stabilité des impulsions [9]. De plus, il est intéressant de noter que différents travaux ont montré que le blocage des phases par pompage synchrone ne permet pas d'obtenir des impulsions aussi brèves et stables que celles délivrées par des lasers à modes couplés par absorbant saturable. Ainsi avec une même caméra et dans des conditions expérimentales identiques, les résolutions temporelles mesurées avec des lasers respectivement à couplages de modes actif et passif étaient de 10 et $\approx 1$ ps $[12,13]$.

Les performances dynamiques du système (caractérisées par le rapport entre les intensités maximale et minimale décelables) sont illustrées sur la figure 5 . La comparaison des distributions de l'impulsion lumineuse avec et sans balayage fait apparaittre l'importance du profil de la fente statique sur les "pieds " de l'histogramme correspondant à l'évolution temporelle de l'impulsion lumineuse; une dynamique effective de $10^{3}$ peut être obtenue aisément, valeur notablement plus élevée que celle que l'on mesure en impulsions uniques [3].

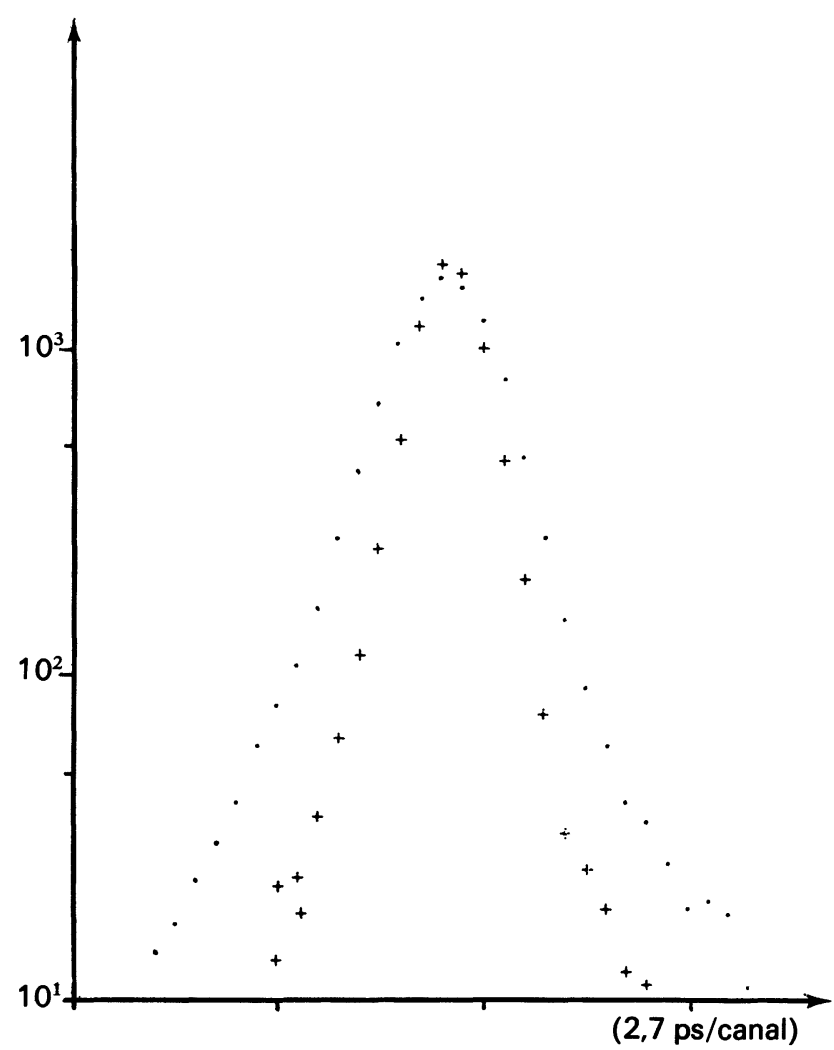

Fig. 5. - Profils statique $\left(+++, l_{1 / 2}^{\mathrm{f}}=9 \mathrm{ps}\right)$ et dynamique $\left(\bullet \bullet \bullet, l_{1 / 2}^{\mathrm{d}}=15 \mathrm{ps}\right)$ de l'impulsion lumineuse.

[Static and dynamic profiles.]

La caméra munie d'un amplificateur accordé à $100 \mathrm{MHz}$ et associée à un laser Nd:YAG continu à modes couplés (Quantronix) a permis de déterminer la largeur, égale à $62 \mathrm{ps}$, des impulsions de $530 \mathrm{~nm}$ obtenues avec un doubleur de fréquence à cristal de KTP à rendement élevé (Fig. 6).

Le montage de la figure 7 diffère de celui décrit précédemment (Fig. 2) par la manière dont est initialisée la synchronisation de la caméra : le signal de l'oscillateur $(40 \mathrm{MHz})$ de l'unité de contrôle (CU) (Spectra Physics) est doublé en fréquence (FD) et appliqué aux plaques de déviation. Ce procédé permet 


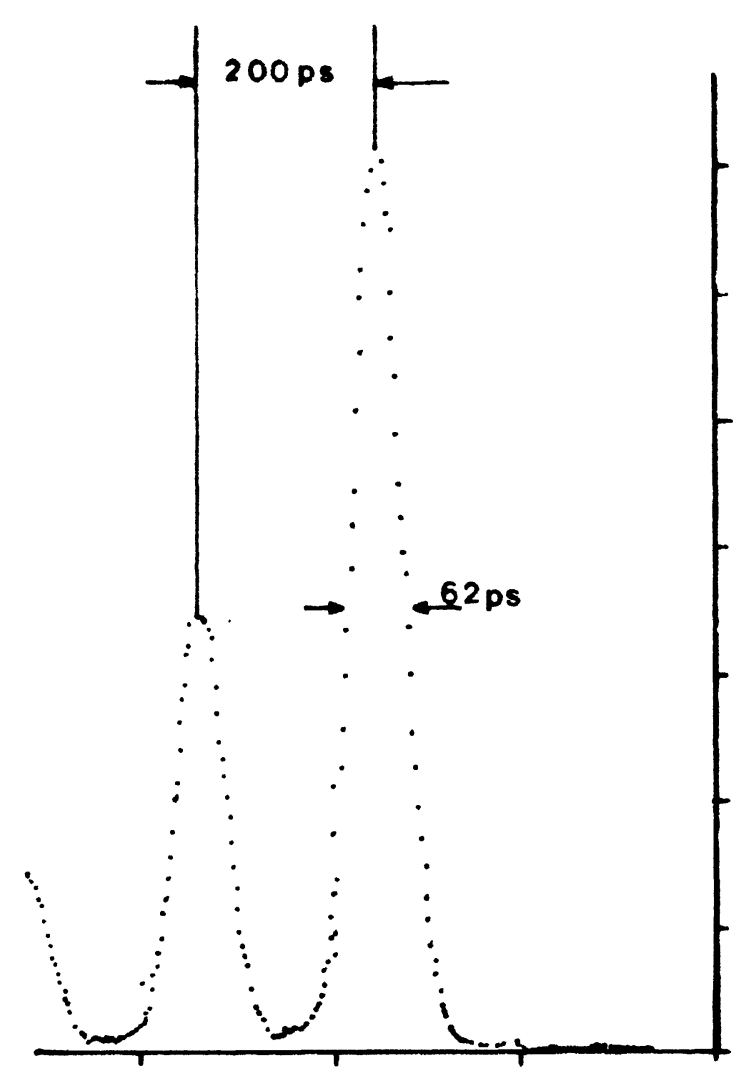

Fig. 6. - Courbe relative aux impulsions délivrées par un laser continu Nd:YAG à modes couplés (Quantronix, $100 \mathrm{MHz})$, après doublage de fréquence $(\lambda=530 \mathrm{~nm})$ : $l_{1 / 2}^{\mathrm{d}}=62 \mathrm{ps}$ (montage expérimental analogue à celui de la figure 2).

[Pulse shape of $530 \mathrm{~nm}$ light pulses of $\mathrm{cw} \mathrm{Nd:YAG} \mathrm{mode}$ locked laser (Quantronix).]

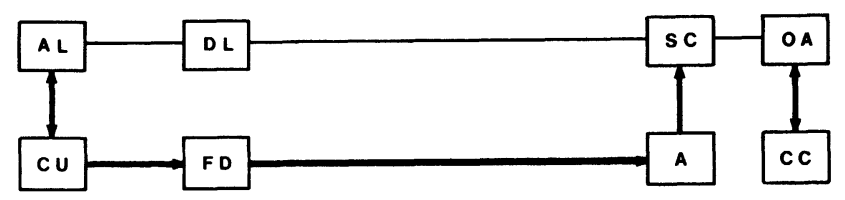

Fig. 7. - Synchronisation de la déflexion sinusoïdale (80 $\mathrm{MHz}$ ) par l'intermédiaire du signal de l'oscillateur (40 MHz) et du doubleur électronique de fréquence (FD).

[Synchroscan deflection by $40 \mathrm{MHz}$ oscillator.]

de relever commodément et indépendamment du faisceau d'excitation les déclins de fluorescence avec une légère perte de résolution (Fig. 8).

Il peut également être utilisé (Fig. 9) lorsqu'au laser à colorant $(\mathrm{DL})$ on associe un dispositif de décharge de cavité (CD) (Spectra Physics), ce qui conduit à des impulsions lumineuses d'énergie plus élevée mais présentant un élargissement de leur durée. Pour une fréquence de répétition de $4 \mathrm{MHz}$, l'énergie par impulsion s'élève à environ $20 \mathrm{~nJ}$ correspondant à une puissance crête voisine de $1 \mathrm{~kW}$; ces valeurs en mode de pompage synchrone $80 \mathrm{MHz}$ sont estimées à $1,5 \mathrm{~nJ}$

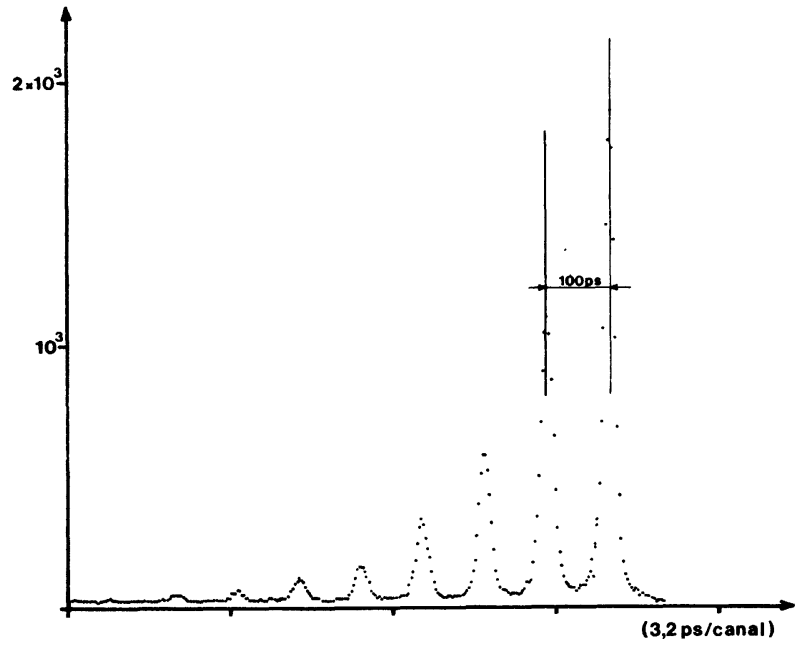

Fig. 8. - Histogramme des impulsions lumineuses du laser à colorant à modes bloqués $(80 \mathrm{MHz})$ obtenu avec le dispositif de la figure 7. (A comparer à la figure $3 a$.)

[Performances obtained with the arrangement of figure 7.]

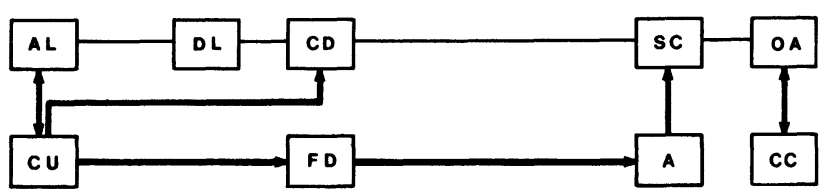

Fig. 9. - Modification du montage de la figure 7 pour son utilisation avec un système de décharge de cavité (CD; S-P 344) : balayage sinusoïdal à $80 \mathrm{MHz}$ et taux de répétition des impulsions laser $4 \mathrm{MHz}$.

[Synchroscan deflection (Fig. 7) and $4 \mathrm{MHz}$ light pulses of a cavity dumped system.]

et $300 \mathrm{~W}$. Pour l'excitation des molécules dans le proche ultra-violet, l'adjonction d'un cristal doubleur de fréquence est indispensable. Le rendement de conversion, faible $(\approx 1 \%)$ pour des puissances crête modérées, étant d'autant plus élevé que la puissance est grande, il est préférable, pour une meilleure sensibilité de détection d'utiliser les impulsions à $4 \mathrm{MHz}$ plutôt que celles du train à $80 \mathrm{MHz}$. La résolution temporelle est alors de $30 \mathrm{ps}$ environ (Fig. 10).

Pour les différents montages (Figs. 2, 7 et 9), la déflexion spatiale est linéaire sur $3 \mathrm{~cm}$ à la sortie de l'amplificateur de brillance (en relation avec la tension $V_{1 / 2}$ de la ddp sinusoïdale de la Fig. 1) et correspond à une plage linéaire temporelle de $1,5 \mathrm{~ns}$ environ.

\subsection{Déflexion par RAMPe De balayage déClen-} CHÉE. - Les caméras munies de circuits de balayage déclenché sont indispensables dans des expériences où les impulsions à analyser sont émises au coup par coup et en régime périodique ou aléatoire. Les distorsions résultant des effets de retour de balayage (Fig. 1) et d'autant plus manifestes que la durée du signal est voisine de la période de la tension de synchronisation peuvent être supprimées avec des générateurs 


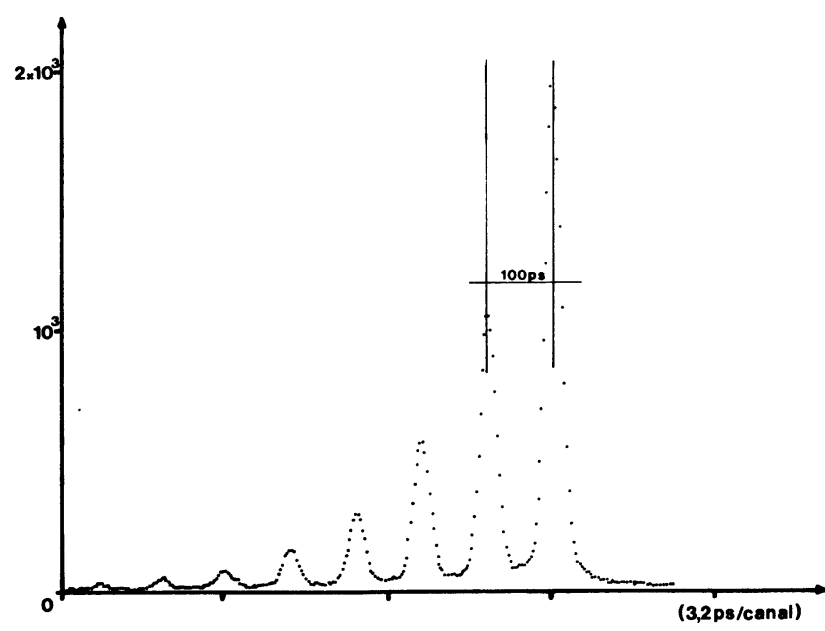

Fig. 10. - Distribution temporelle obtenue avec le dispositif de la figure 9 pour une fréquence de répétition de $4 \mathrm{MHz}$ des impulsions lumineuses délivrées par le laser à décharge de cavité.

[Time resolution obtained with the set-up of figure 9.]

de rampe. L'appareillage de la figure 11 représentant schématiquement un laser picoseconde $(\mathrm{AL}, \mathrm{DL})$ à extracteur d'impulsions (CD) peut être utilisé en mode récurrent (fréquence de répétition maximale $800 \mathrm{kHz}$ ) et en impulsion unique. En effet, l'adjonction d'une cellule de Pockels (PC) dont l'ouverture est commandée par un générateur à transistors d'avalanche (AC) permet la sélection d'une impulsion unique dans le train périodique de $200 \mathrm{~Hz}-800 \mathrm{kHz}$. Remarquons qu'il est également possible de déclencher l'ouverture de la cellule de Pockels avec une fréquence variable $(\$ 30 \mathrm{kHz})$ [14] à partir de la console de commande (CC) à travers une porte électronique $(\mathrm{G})$. Le taux de répétition du balayage est prédéterminé par l'unité de contrôle (CU) et son déclenchement est initialisé par le signal délivré par la diode (D) qui reçoit une partie du faisceau lumineux par l'intermédiaire du miroir (M). Celui-ci peut être situé soit directement après le laser à argon, soit après le laser à colorant à décharge de cavité. Dans les expériences à coups uniques, un obturateur (S) est placé devant la photocathode (SC) pour éviter un éblouissement de la caméra dû à la lumière parasite du faisceau.

Les histogrammes expérimentaux 12a et b ont été enregistrés avec une fréquence de répétition de $400 \mathrm{kHz}$, le déclenchement du circuit de balayage étant effectué par les impulsions lumineuses du laser à colorant, pour deux réglages de la longueur de la cavité du laser à colorant donnant lieu à des largeurs à mi-hauteur des corrélogrammes de 25 et 12,5 ps. Les distributions 12a et $b$ présentent des largeurs identiques ( $24 \mathrm{ps}$ ), mais des distorsions de forme très importantes : la dégradation de la résolution peut être caractérisée par la diminution du rapport pic/vallée qui passe de 20 à 4 . Pour expliquer l'origine de ces observations, on a relevé, pour les deux réglages du laser, les courbes dues à un

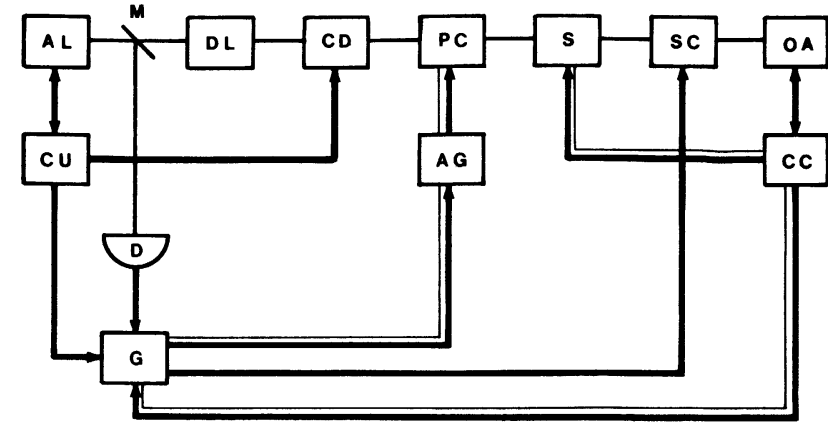

Fig. 11. - Montage utilisé avec un dispositif à décharge de cavité $(C D)$ en régime de balayage déclenché au moyen des impulsions lumineuses du laser à argon $(\mathrm{AL})$. $\mathrm{G}$ : porte électronique; $\mathrm{AG}$ : générateur à transistors d'avalanche; PC : extracteur d'impulsion à cellule de Pockels; $\mathbf{S}$ : obturateur électromécanique. - (trait fin) : voie optique, - (trait épais) : liaisons électroniques, $=$ (double trait) : liaisons électroniques pour l'utilisation de (PC).

[Triggered deflection system and cavity dumped operation.]

éclairement par une seule impulsion (Fig. 13a et b). D'une façon générale, on observe des fluctuations en forme, en durée et en position des impulsions, mais avec une prépondérance marquée dans le cas (Fig. 13b) où le corrélogramme est le plus étroit (12,5 ps), ce qui correspond à une cavité raccourcie par rapport à celle donnant le meilleur rapport pic/vallée en mode répétitif et le corrélogramme le plus large ( 24 ps). Des instabilités apparaissent lorsque la longueur de la cavité est encore diminuée alors que si elle est supérieure à la longueur optimale, une augmentation de la durée des impulsions est évidente. Il faut souligner (Fig. 12a) l'excellente linéarité du balayage ainsi que la valeur élevée de la dynamique $\left(>10^{2}\right)$, nettement mises en évidence en représentation semi-logarithmique.

De ces données, il découle que la résolution instrumentale de 24 ps est essentiellement limitée par les fluctuations provenant du laser et non de l'électronique de déclenchement (cf. discussion $\S 3.1$ ) et que la caractérisation des impulsions lumineuses par un autocorrélateur est peu satisfaisante. Cette dernière technique présente deux inconvénients majeurs : d'une part la forme du flash lumineux n'est pas directement liée à la courbe enregistrée [1], d'autre part l'emploi de moyenneur (un point de la courbe est la moyenne d'un grand nombre de mesures) ne peut donner lieu à l'observation de fluctuations de la forme des impulsions [15]. Cependant, l'analyse du corrélogramme fournit des données "en moyenne" sur les caractéristiques et la nature spectrale des impulsions lumineuses.

Le résultat de la figure $12 \mathrm{c}$ a été obtenu également avec le montage de la figure 11, sans adjonction de la cellule de Pockels (taux de répétition de $400 \mathrm{kHz}$ ), le balayage étant déclenché par les impulsions du laser à argon et dans les conditions de réglage optimal 


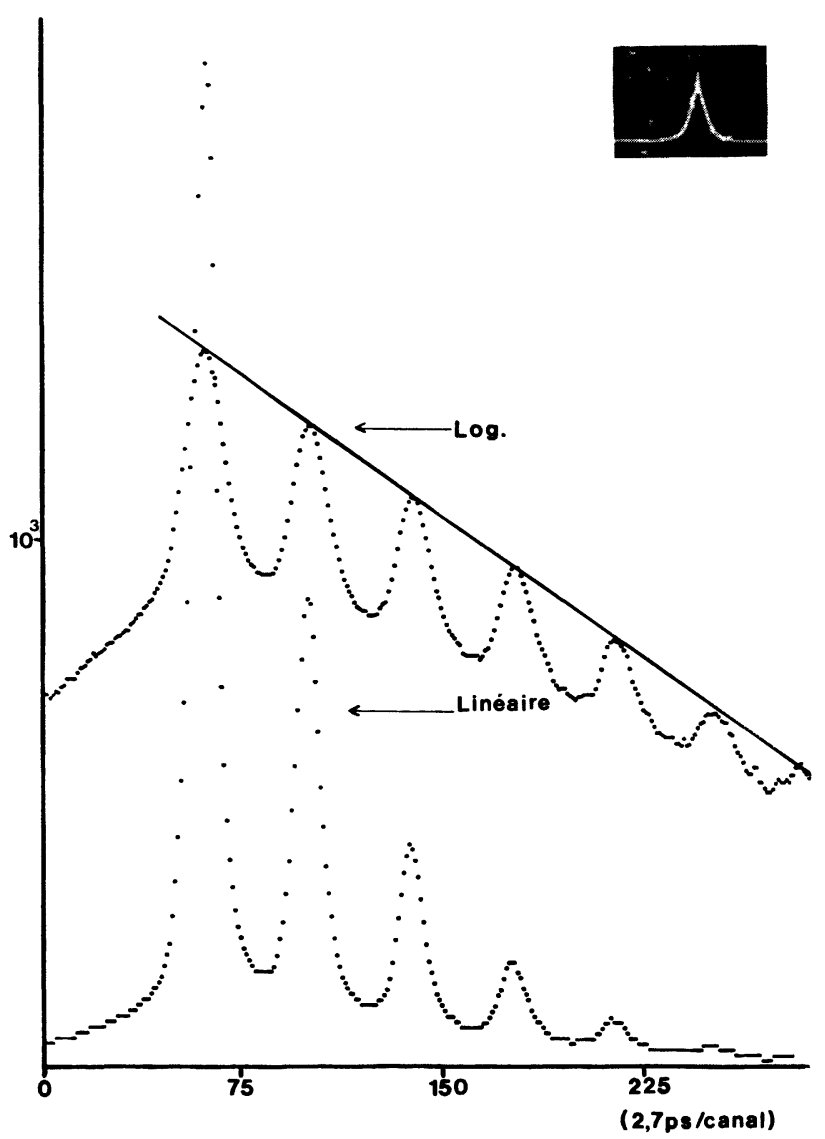

a)

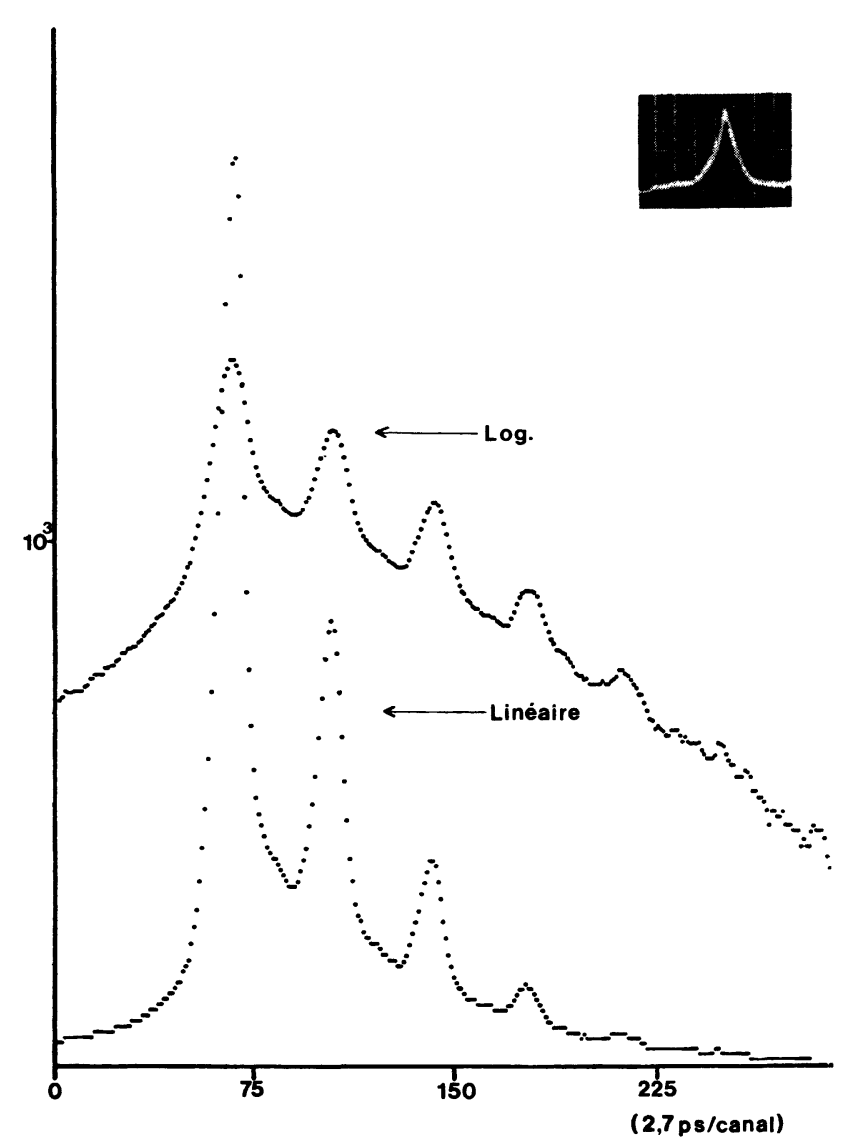

b)

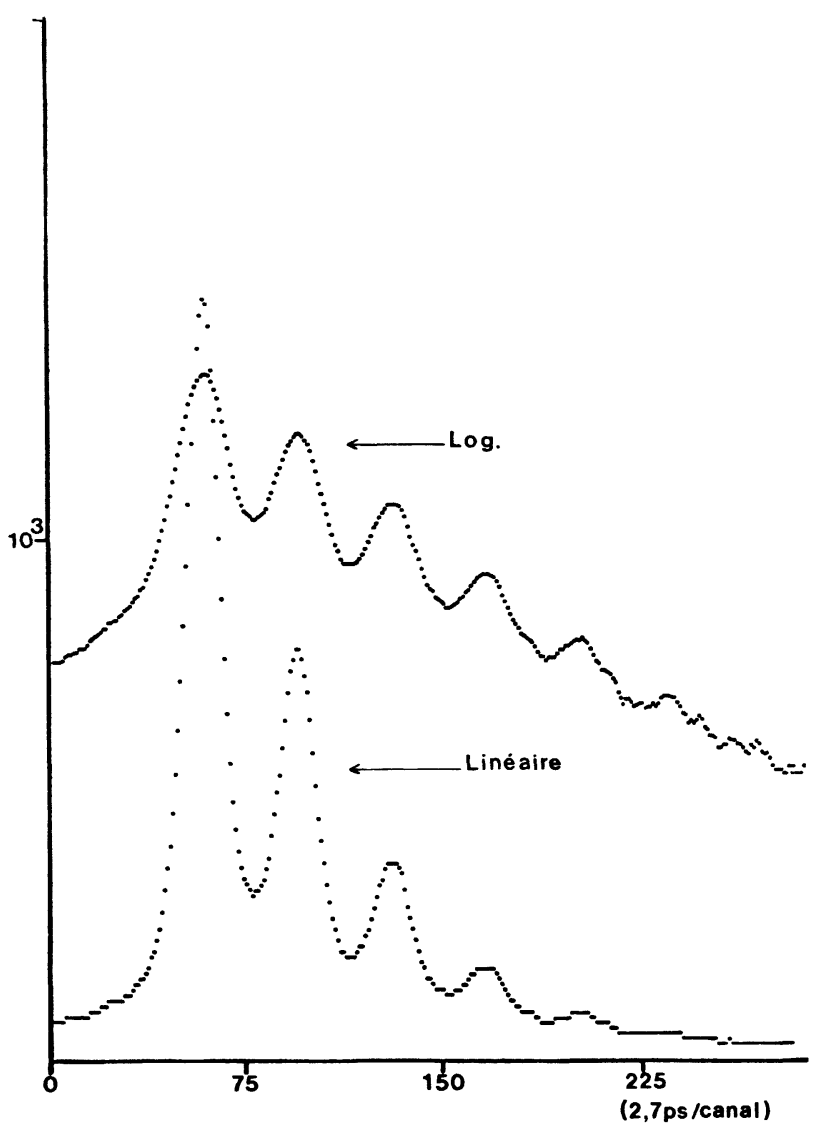

c)

Fig. 12. - Histogrammes obtenus en régime récurrent déclenché $(400 \mathrm{kHz})$ et corrélogrammes correspondants. (a) et (b) : déclenchement par les impulsions du laser à colorant; (a) : réglage optimal de la cavité $\left(l_{1 / 2}^{\mathrm{h}}=24 \mathrm{ps}\right.$, $\left(l_{1 / 2}^{\mathbf{c}}=25 \mathrm{ps}\right) ; \quad$ (b) : configuration cavité raccourcie $\left(l_{1 / 2}^{\mathrm{h}}=24 \mathrm{ps}, l_{1 / 2}^{\mathrm{c}}=12,5 \mathrm{ps}\right)$; (c) déclenchement par les impulsions du laser à argon et réglage optimal de la cavité $\left(l_{1 / 2}^{\mathrm{h}}=38 \mathrm{ps}\right)$.

[Time fluctuations between argon ion and dye lasers.]

de la longueur de la cavité : l'élargissement de la largeur de la fonction de réponse ( $38 \mathrm{ps)} \mathrm{est} \mathrm{apparemment} \mathrm{dû}$ aux fluctuations temporelles entre les impulsions lumineuses des lasers argon (AL) et à colorant (DL, CD); des ajustements des longueurs de cavité des deux lasers ne conduisent pas à une amélioration de la résolution temporelle.

La caractérisation des impulsions de longueur d'onde de $530 \mathrm{~nm}$ d'un laser Nd:YAG à modes couplés et déclenché (Fig. 14) a été effectuée à l'aide d'un tube à fente EEV P855. Après conversion par un cristal de KTP (FFD), le train (S1) d'impulsions infra-rouges (séparées de $10 \mathrm{ns)} \mathrm{d'enveloppe} \mathrm{gaussienne} \mathrm{de} \mathrm{durée}$ $200 \mathrm{~ns}$ et d'énergie $1 \mathrm{~mJ}$, donne naissance à un train (S2) d'environ 50 ns et d'énergie voisine de $700 \mu \mathrm{J}$. Le sélecteur d'impulsions (TS) $[16,17$, relié à l'unité de contrôle (CU) assurant le couplage des modes et le fonctionnement en régime déclenché du laser, fournit un signal (S3) à fréquence de répétition ajustable 

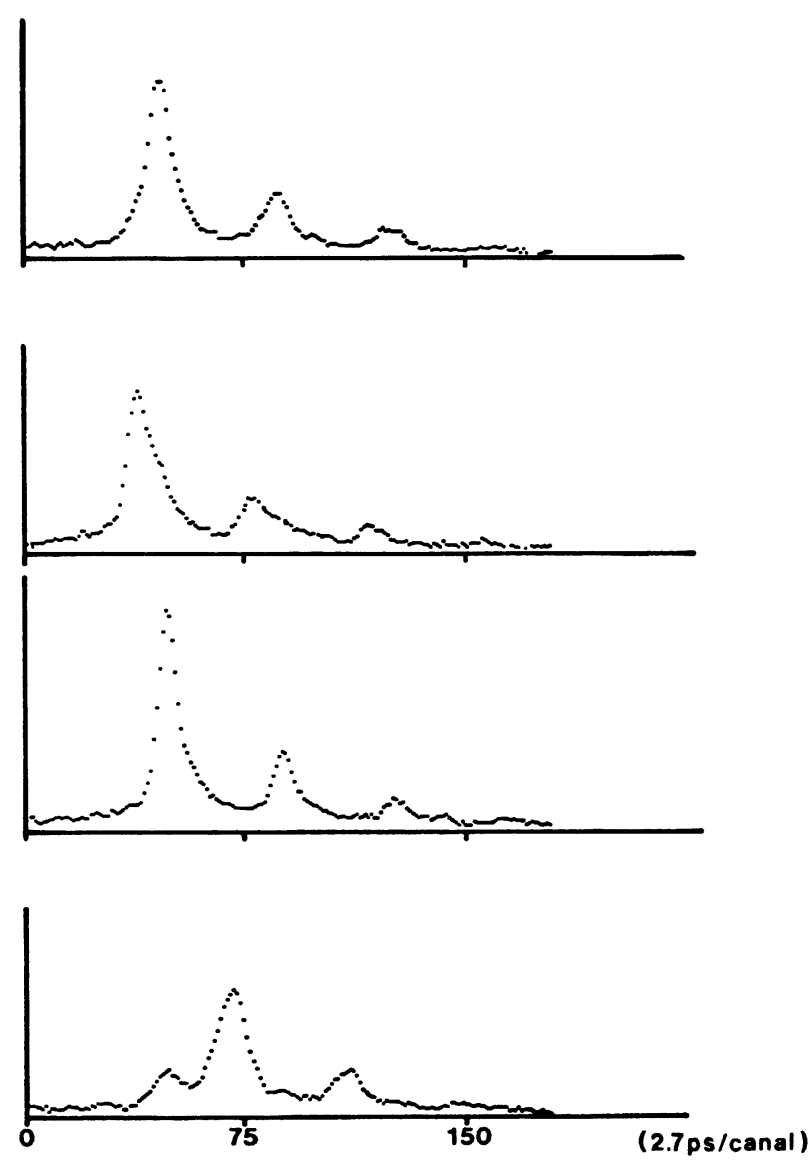

a)

Fig. 13. - Histogrammes à " coups uniques" (montage Fig. 11)(a) Réglage optimal de la cavité, (b) Cavité raccourcie.

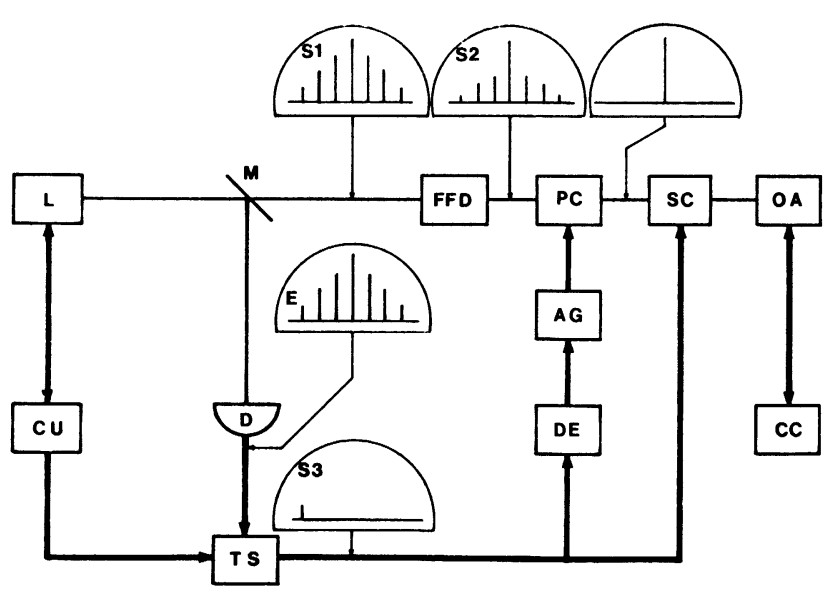

Fig. 14. - Montage d'utilisation d'un laser Nd:YAG (Quantronix) à modes couplés et déclenché ( $Q$-switch) avec une caméra à balayage de fente fonctionnant en régime déclenché. L : laser Nd:YAG; CU : unité de contrôle; FFD : doubleur de fréquence; DE : ligne à retard variable; TS : dispositif électronique pour la sélection d'une impulsion.

[Experimental device using a cw mode locked and $Q$ switched Nd : YAG laser.]
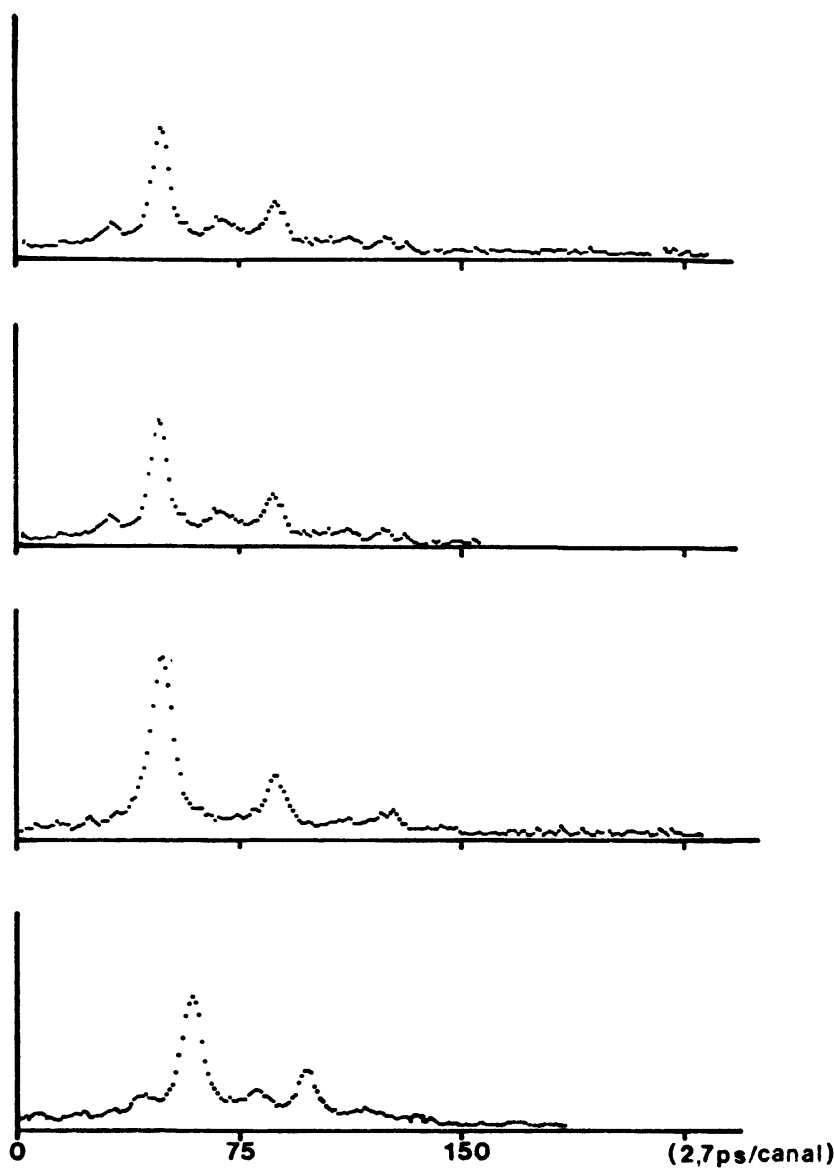

b)

[Single shot measurements illustrating the influence of the cavity length.]

$(0-1 \mathrm{kHz})$ en relation avec l'éclair $E$ en avance de $n \times 10$ ns par rapport à l'impulsion d'intensité maximale du train (S1). Convenablement retardé (DE), le signal (S3) commande l'ouverture de l'obturateur de Pockels (AG, PC) qui laisse passer l'impulsion d'intensité maximale du train (S2). Son analyse temporelle est réalisée par (SC), le balayage étant initialisé par (S3). De l'histogramme (Fig. 15) relevé avec un taux de $500 \mathrm{~Hz}$, on déduit une durée des impulsions d'environ $80 \mathrm{ps}$.

\section{Application à la mesure des déclins de fluorescence.}

Pour illustrer les performances temporelles que l'on peut atteindre avec la caméra à balayage de fente dans les expériences de photoluminescence, on a reporté sur la figure 16 les courbes de décroissance de l'émission de solutions dégazées de pinacyanol $\left(10^{-5} \mathrm{M}\right)$ dans le propanol relevées à l'aide du montage de la figure 2 et ce pour différentes températures de l'échantillon. On a comparé (Fig. 17) les histogrammes expérimentaux (traits pleins) à ceux obtenus par convolution de la fonction de réponse instrumentale et d'une 


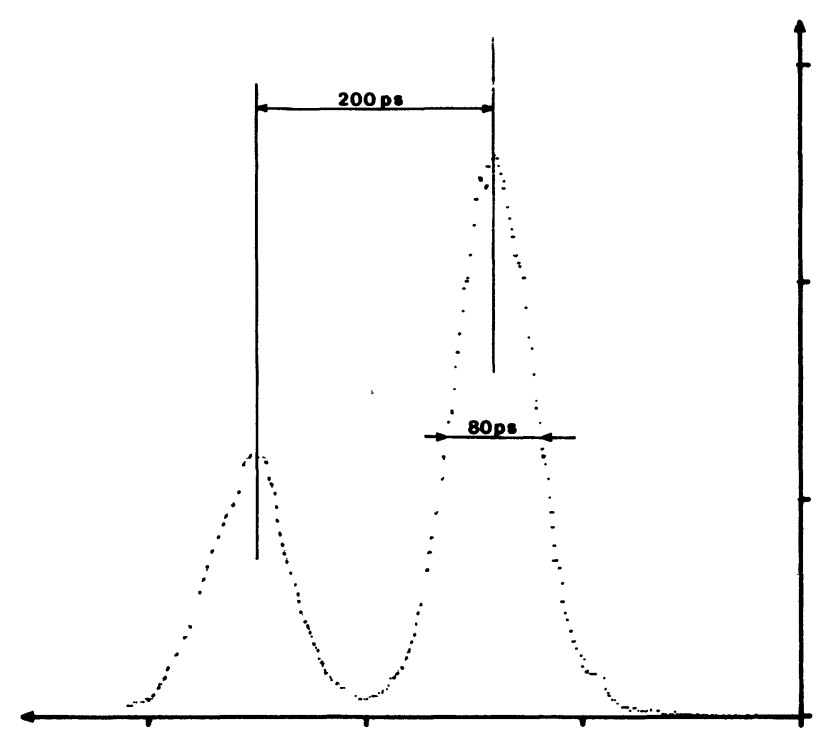

Fig. 15. - Histogramme du signal lumineux $(\lambda=530 \mathrm{~nm})$ d'intensité maximale extraite du train d'impulsions d'un $\mathrm{Nd}$ :YAG à modes couplés et déclenché.

[Pulse shape at $530 \mathrm{~nm}$ of the Nd : YAG laser (Fig. 14).]

loi de déclin exponentielle de constante de temps variant entre 10 et $150 \mathrm{ps}$. On constate une très bonne superposition des courbes expérimentales et calculées, et la précision de l'évaluation des durées de vie de l'état excité du pinacyanol est estimée meilleure que $10 \%$

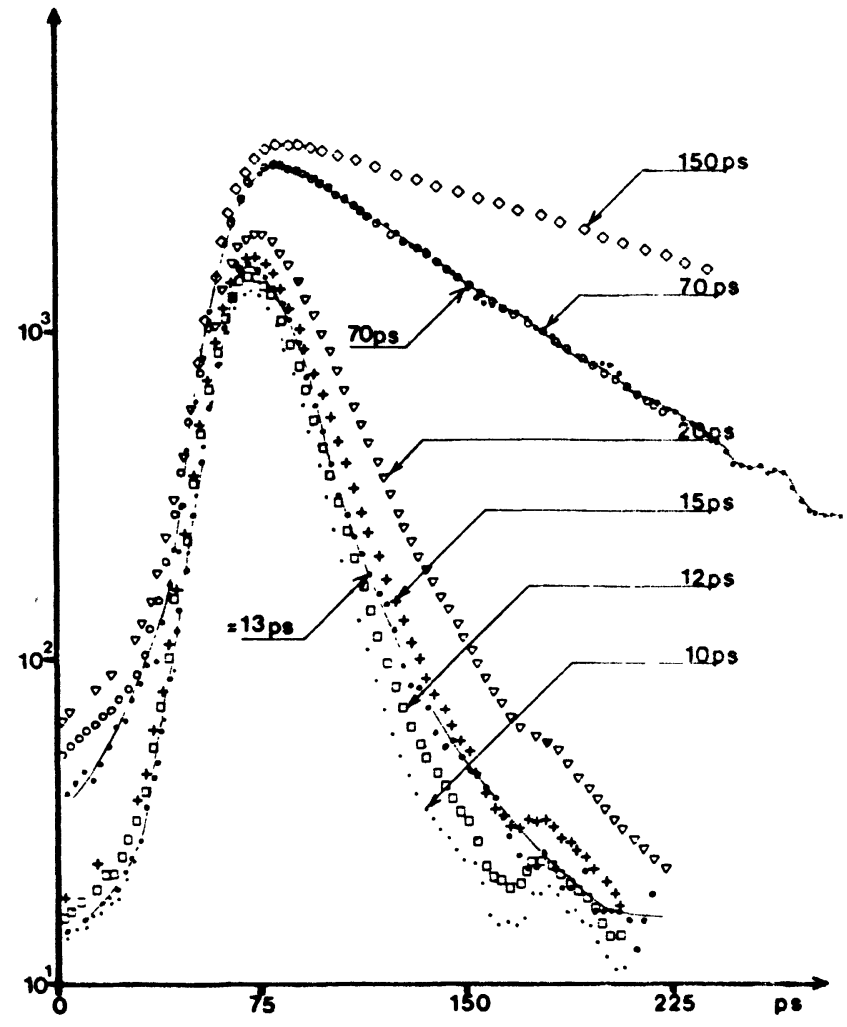

Fig. 17. - Ajustement des courbes calculées et expérimentales (trait plein).

[Experimental and theoretical fits.]

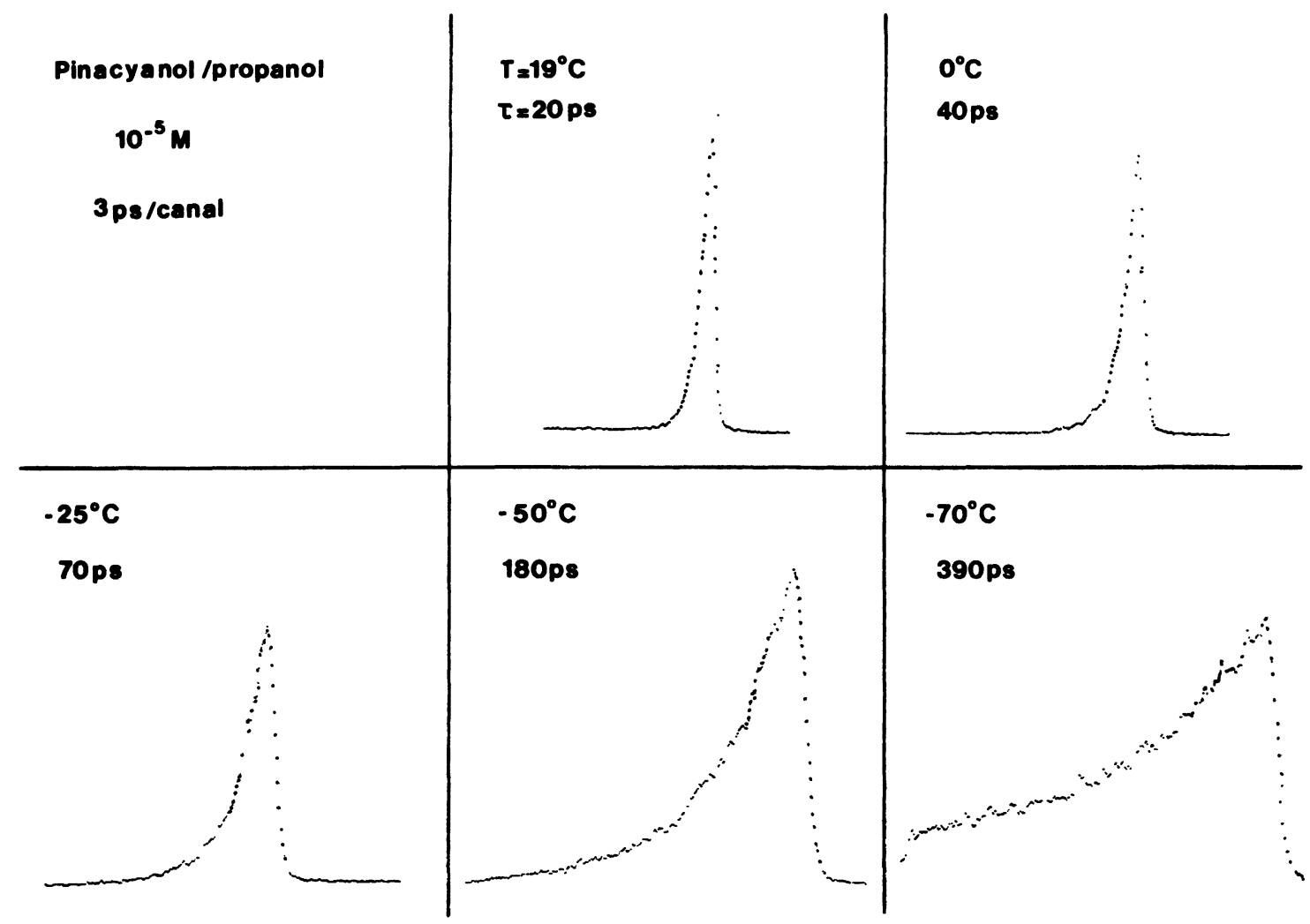

Fig. 16. - Courbes de déclin de la fluorescence d'une solution de pinacyanol/propanol à différentes températures. [Fluorescence decay of pinacyanol.] 
Annexe.

Les proprietés d'un générateur de balayage sont généralement caractérisées par les linéarités différentielle et intégrale du système de déflexion. Pour une fonction de transfert sinusoïdale $\xi=\sin \varphi$, $\xi$ et $\varphi$ étant des variables réduites égales à $x / x^{0}$ et $2 \Pi t / T$ où $T$ est la période, $x_{0}$ et $x$ représentent l'élongation maximale et celle à l'instant $t$. La linéarité intégrale $\mathcal{L}_{\mathrm{i}}(\xi)$ définie par

$$
\mathcal{L}_{i}(\xi)=\frac{\sin \varphi}{\varphi} \leqslant 5 \%
$$

conduit à une excursion spatiale $|\xi| \leqslant 1 / 2$ et la linéarité différentielle $\mathcal{L}_{d}(\xi)$ est donnée par l'histogramme de la densité spatiale de $\xi$ pour une loi uniforme des phases, ce qui, expérimentalement, consiste à enregistrer la distribution spatiale de l'intensité lumineuse sur l'écran luminophore consécutive à une impulsion d'excitation rectangulaire.

D'une manière générale, si $\xi=g(\varphi)$ où $g$ est la fonction de transfert et si $f_{\Phi}(\varphi)$ est la densité de la phase, la loi de probabilité $f_{z}(\xi)$ de $\xi$ est donnée par

$$
f_{\Xi}(\xi)=\sum_{i} \frac{f_{\Phi}\left(\varphi_{i}\right)}{\left|g^{\prime}\left(\varphi_{i}\right)\right|}
$$

où $\varphi_{i}$ est la solution de l'équation $\xi=g(\varphi)$ et $g^{\prime}$ la dérivée de $g$.

D'où, par application de la relation précédente au

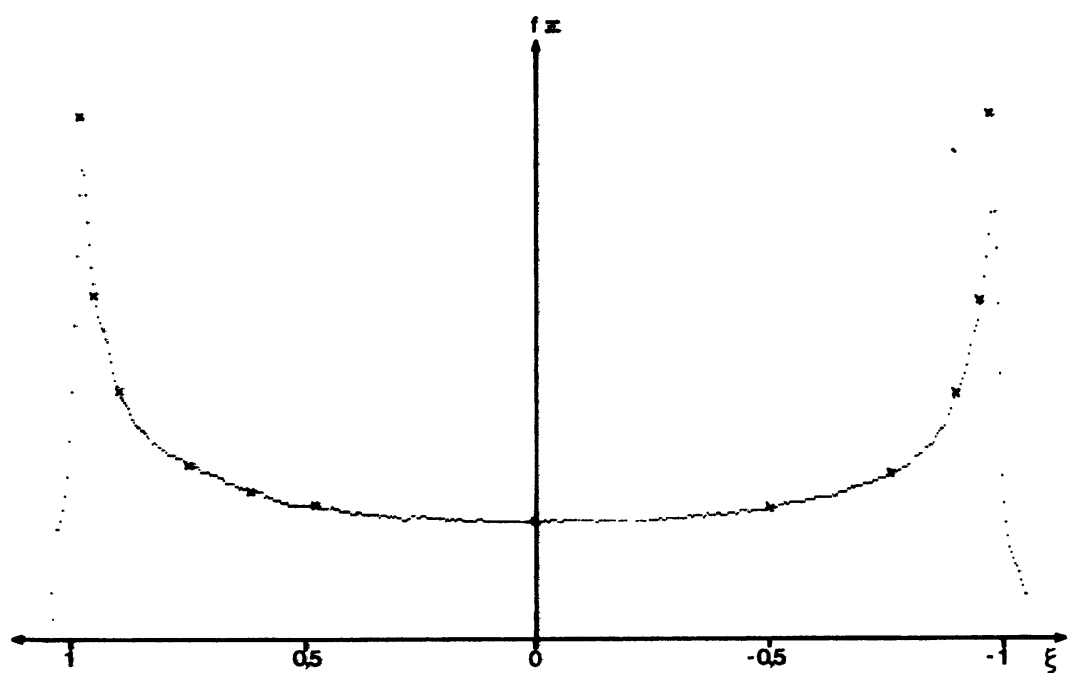

Fig. 18. - Linéarités différentielles $\mathcal{L}_{d}(\xi)$ expérimentale $(\bullet \bullet \bullet \bullet)$ et calculée $(\times \times \times)$ pour un balayage sinusoïdal. [Calculated and experimental differential linearities.]

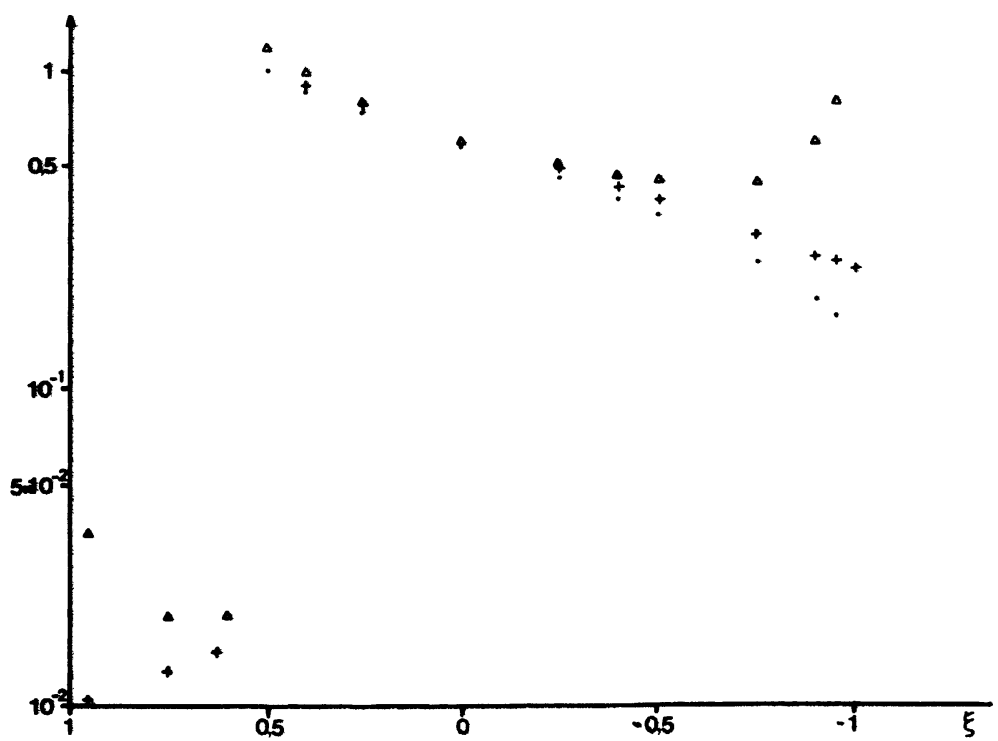

Fig. 19. - Histogrammes calculés pour $\alpha=1, \theta=5 \Pi / 6, \bullet \bullet \bullet \bullet h_{1}(\xi),+++++h(\xi), \times \times \times \times f_{\Xi}(\xi)$.

[Probability density curve for an exponential decay.] 
cas d'une fonction sinusoïdale pour $g(\varphi)$

avec

$$
\mathfrak{L}_{\mathrm{d}}(\xi)=\frac{1}{\Pi} \frac{1}{\left(1-\xi^{2}\right)^{1 / 2}}
$$

$$
f_{\Phi}(\varphi)=\frac{1}{2 \Pi} \quad 0<\varphi<2 \Pi .
$$

Sur la figure 18 sont représentés l'histogramme calculé $\mathfrak{L}_{\mathrm{d}}(\xi)$ et la courbe expérimentale relevée à l'aide de l'analyseur multicanal optique placé à la sortie du tube de déflexion pour une tension sinusoïdale de balayage de fréquence $80 \mathrm{MHz}$ et d'amplitude crête voisine de $2 \mathrm{kV}$ et pour un éclairemment stationnaire de la fente de la photocathode. Pour une impulsion lumineuse incidente correspondant à un déclin de fluorescence exponentiel (voir Fig. 1), c'est-à-dire :

$$
\begin{aligned}
f_{\Phi}(\varphi)=\mathrm{e}^{-\alpha(\varphi-\theta)} & \alpha>0 \\
\varphi-\theta & \geqslant 0
\end{aligned}
$$

la répartition de l'intensité sur l'écran luminophore sera donnée par

$f_{\xi}(\xi)=\frac{1}{\left(1-\xi^{2}\right)^{1 / 2}} \mathrm{e}^{-\alpha(\arcsin \xi-\theta)}=\frac{1}{\left(1-\xi^{2}\right)^{1 / 2}} h(\xi)$

où $h(\xi)$ peut être défini comme l'image de $f_{\Phi}(\varphi)$ due à la fonction de transfert sinusoïdale. Sur la figure 19 , on a représenté les histogrammes $h(\xi)$ et $f_{\bar{\xi}}(\xi)$ calculés pour $\theta=\frac{5 \Pi}{2}$ et $\alpha=1$, valeur qui traduit une durée de déclin égale à $T / 2 \Pi$. A titre indicatif, on a également reporté la fonction $h_{1}(\xi)$ qui représente ce que l'on obtiendrait après une demi-période de balayage $\left(\frac{5 \Pi}{6} \leqslant \varphi \leqslant \frac{3 \Pi}{2}\right)$ et après correction par la linéarité différentielle. On constate une excellente linéarité globale pour $|\xi| \leqslant 0,4$, la constante de déclin mesurée étant égale à la valeur théorique à mieux que $5 \%$ près. Les courbes de la figure 20 obtenues avec $\alpha=0,5$ $(\theta=5 \Pi / 6)$ mettent en évidence de façon nette les effets du retour du balayage lorsque la constante de décroissance est plus grande, influence qui sera d'autant plus marquée que la durée du signal lumineux sera voisine de la demi-période de la tension de déflexion.

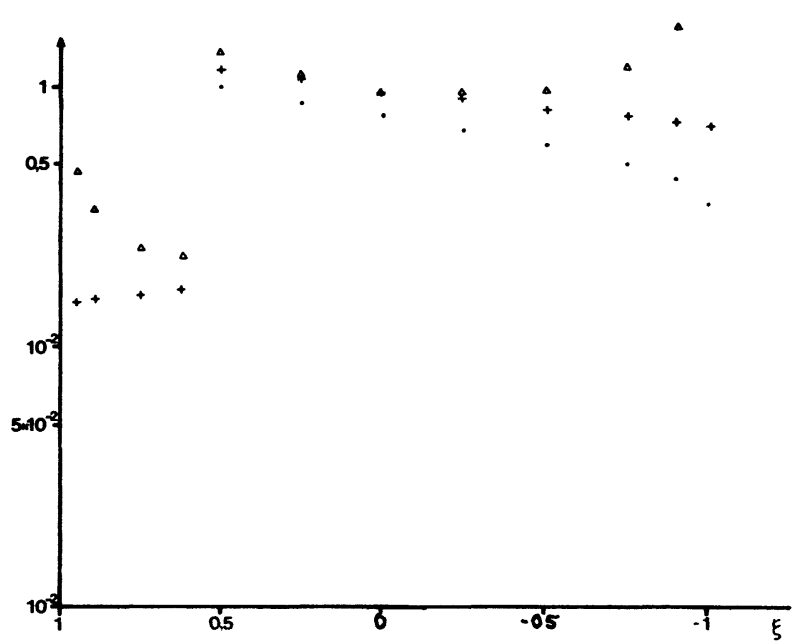

Fig. 20. - Histogrammes calculés pour $\alpha=0,5, \theta=5 \Pi / 6$, $\bullet \bullet \bullet \bullet \bullet h_{1}(\xi),+++++h(\xi), \times \times \times \times f_{\xi}(\xi)$.

[Probability density curve for an exponential decay.]

\section{Bibliographie}

[1] Ultrashort Light Pulses, éd. S. L. Shapiro (Springer Verlag, Berlin), 1977.

[2] Picosecond Phenomena I, II et III (Springer Verlag, Berlin), 1978, 1980, 1982.

[3] Heisel, F., Miehé, J. A., Sipp, B., Ann. Phys. Fr. 4 (1979) 331.

[4] Campillo, A. J., Shapiro, S. L., IEEE J. Quantum Electron. QE 19 (1983) 585.

[5] Adams, M. C., Bradley, D. J., Sibbett, W., Taylor, J. R., Chem. Phys. Lett. 66 (1979) 428.

[6] Heisel, F., MiehÉ, J. A., SipP, B., Rev. Sci. Instrum. 52 (1981) 992.

[7] Geist, P., Heisel, F., Martz, A., Miehé, J. A., Optics Commun. 45 (1983) 17.

[8] Adv. Electron. Electron Phys.; Vol. 33B (1972); Vol. 52 (1979).

[9] Cavailler, C., Fleurot, N., Mens, A., Knispel, G.,
Miehé, J. A., Proc. 8th Symposium on Photoelectronic Image Devices, London, 1983, SPIE.

[10] Sibbett, W., Sleat, W. E., Taylor, J. R., Willson, J. P., Proc. 15th Int. Congress on High Speed Photography and Photonics, San Diego, 1982, SPIE, Vol. 348.

[11] Shapiro, S. L., Cavanagh, R. R., Stephenson, J. C., Optics Lett. 6 (1981) 470.

[12] Mourou, G., Sizer, T., Optics Commun. 41 (1982) 47.

[13] Sibbett, W., Proc. 15th Int. Congress on High Speed Photography and Photonics, San Diego 1982, SPIE 348.

[14] MarTZ, A., Rapport Interne.

[15] Van Stryland, E. W., Optics Commun. 31 (1979) 93.

[16] Cunin, B., Miehé, J. A., Sipp, B., Thébault, J., Rev. Sci. Instrum. 47 (1976) 6.

[17] GeIST, P., Rapport interne. 